\title{
EFEITO DAS CONDIÇÕES AMBIENTAIS NO NÍVEL DE RUÍDO EMITIDO POR LEITÕES
}

\author{
KESIA O. DA SILVA-MIRANDA ${ }^{1}$, GISELLE BORGES ${ }^{2}$, VINÍCIUS L. DE C. \\ MENEGALE ${ }^{3}$, IRAN JOSE O. DA SILVA ${ }^{4}$
}

\begin{abstract}
RESUMO: Objetivou-se, com este trabalho, avaliar a influência das variáveis ambientais nos níveis de ruídos emitidos por suínos e quantificar as faixas em $\mathrm{dB}$ comparativamente às condições de conforto térmico estabelecidas pela literatura. $\mathrm{O}$ experimento foi conduzido em câmara climática, onde foram alojados cinco leitões em fase de creche, submetidos à variação na temperatura ambiente de $20^{\circ} \mathrm{C}$ a $38^{\circ} \mathrm{C}$ e umidade relativa de $50 \%$ a $80 \%$. Decibelímetros foram instalados para o registro dos níveis de ruídos e sensores dataloggers para os dados de temperatura e umidade relativa. O nível de atividade foi utilizado para quantificar a movimentação dos animais por intermédio de análise de imagens. Análises de correlação e regressão foram aplicadas nos dados para análise estatística. As variáveis ambientais influenciam na emissão de ruídos pelos leitões quando expostos a diferentes condições térmicas. Os níveis de ruídos foram estabelecidos em faixas de acordo com a condição térmica a que animais foram submetidos. Para a condição de conforto (20 a $\left.23^{\circ} \mathrm{C}\right)$, níveis de ruídos na faixa de 70 a $75 \mathrm{~dB}$; condição de alerta $\left(23\right.$ a $\left.30^{\circ} \mathrm{C}\right)$, níveis de ruídos na faixa de 60 a $70 \mathrm{~dB}$ e para condição de estresse térmico (acima de $30^{\circ} \mathrm{C}$ ), na faixa de 55 a $60 \mathrm{~dB}$.
\end{abstract}

PALAVRAS-CHAVE: ruído animal; vocalização; temperatura; decibelímetro.

\section{EFFECTS OF ENVIRONMENTAL CONDITIONS ON SOUND LEVEL EMITTED BY PIGLETS}

\begin{abstract}
The objective of this study was to assess the influence of environmental variables on sound level emitted by piglets, and to quantify the sound bands using $\mathrm{dB}$ compared to the conditions of thermal comfort established by the literature. The experiment was carried out at a climatic chamber, where five piglets at nursery stage were housed and submitted to a variation of the ambient temperature ranging from $28^{\circ} \mathrm{C}$ to $38^{\circ} \mathrm{C}$ and relative humidity ranging from $50 \%$ to $80 \%$. Sound level meters were installed to record sound levels, and data loggers to record temperature and relative humidity data. The level of activity was used to quantify the animals' movement by means of image analysis. Correlation and regression analysis were applied in data for statistical analysis. Environmental variables have influence the sound level emitted by piglets when they are exposed to different thermal conditions. The sound levels were established in bands according to the thermal condition in which the animals are submitted. For comfort condition (20 to $23^{\circ} \mathrm{C}$ ), sound level ranges from 70 to $75 \mathrm{~dB}$; for alert condition $\left(23\right.$ to $\left.30^{\circ} \mathrm{C}\right)$, sound level ranges from 60 to $70 \mathrm{~dB}$; and for thermal stress condition (above $30^{\circ} \mathrm{C}$ ), it ranges from 55 to $60 \mathrm{~dB}$.
\end{abstract}

KEYWORDS: animal noise; vocalization; temperature; sound level meter.

\footnotetext{
${ }^{1}$ Engenheira Agrícola, Profa. Doutora do Depto. de Engenharia de Biossistemas, Vice - Coordenadora do NUPEA, ESALQ - USP.

${ }^{2}$ Engenheira Agrícola, Pesquisadora do NUPEA, Depto de Engenharia de Biossistemas - ESALQ-USP, giborges.mg@gmail.com.

${ }^{3}$ Eng. Agronômo, Mestrado em Agronomia - Energia na Agricultura na FCA/UNESP - Botucatu/SP.

${ }^{4}$ Engenheiro Agrícola, Prof. Livre Docente do Depto de Engenharia de Biossistemas, Coordenador do NUPEA, ESALQ - USP.

Recebido pelo Conselho Editorial em: 2-6-2011

Aprovado pelo Conselho Editorial em: 3-2-2012
} 


\section{INTRODUÇÃO}

A avaliação da resposta dos animais aos diferentes níveis de estresse e bem-estar ainda é pouco difundida entre os pesquisadores da área, sendo de extrema importância sua caracterização, pois pode ser um indicativo, caso o ambiente e as condições de manejo não estejam propícios ao animal. Neste contexto, as formas de se avaliar e quantificar o bem-estar do animal em ambiente de produção intensiva vem destacando-se como tema mais importante entre os pesquisadores da área (NÄ̈̈S et al., 2008). Dentre os diferentes mecanismos de avaliação, despontam como metodologia inovadora de indicativo comportamental os registros e os estudos do nível de ruído de um grupo de animais e da vocalização.

Dentre as variáveis ambientais, a temperatura do ar pode afetar a saúde, o bem-estar e a eficiência na produção intensiva de suínos, considerando essencial entender a resposta do animal confinado em relação ao ambiente térmico, a fim de minimizar os efeitos negativos do desconforto térmico (BANHAZI et al., 2008).

A primeira reação de um suíno ao aumento da temperatura é a mudança no comportamento (HUYNH et al., 2005), reduzindo sua atividade e buscando por áreas úmidas. Durante os meses mais quentes, a média diária do ganho e o consumo voluntário de ração estão abaixo do esperado, afetando negativamente a produção de suínos. Em geral, a faixa média de temperatura considerada confortável para suínos na fase de crescimento varia entre 18 e $21^{\circ} \mathrm{C}$ (HUYNH et al., 2004).

Um estudo proposto por BROWN-BRANDL et al. (2001) avaliou os efeitos do estresse por calor em suínos nas fases de crescimento e terminação, em ambiente controlado. Os autores avaliaram a produção de calor dos suínos, a frequência respiratória, a temperatura retal e verificouse aumento de $17-20 \%$ na taxa de produção de calor e que a frequiência respiratória foi o melhor indicador de estresse nos suínos. Contudo, altas temperaturas ambientais afetam os suínos de maneira negativa, ocasionando alterações em sua temperatura retal, superfície da pele e frequência respiratória (MANNO et al., 2005).

Além das técnicas de análise de imagens utilizadas para caracterizar o comportamento animal, frente às situações adversas (PANDORFI et al., 2004; SILVA et al., 2004; RODRIGUES, 2006; BARBOSA FILHO et al., 2007), têm-se buscado métodos que avaliam a resposta direta do animal, tais como estudos dos níveis de ruído emitidos por um grupo de animais, como uma forma de quantificar sua situação em relação ao ambiente térmico.

Técnicas de análise de imagens, estudo de vocalização e níveis de ruído, são métodos não invasivos para avaliar a resposta do animal em situações adversas, pois os dados podem ser registrados sem o estresse, perturbação ou manipulação do animal.

Os sons emitidos pelos animais podem ser monitorados, e suas frequências analisadas para avaliar o estado de saúde dos animais (VAN HIRTUM \& BERCKMANS, 2004). Segundo BOISSY et al. (2007), a análise do comportamento e de vocalizações dos animais serão os indicadores promissores para avaliar respostas positivas dos animais estudados tanto em laboratório quanto em ambiente de produção intensiva.

Destaca-se o nível de ruído na avaliação do grupo de animais, cujos dados são expressos na escala de decibéis e a vocalização utilizada para o caráter individual do animal (SILVA \& NÄÄS, 2006), porém trabalhos avaliando os níveis de ruído emitido por leitões foram conduzidos em ambiente de produção intensiva, no qual não conseguiram controlar as condições do ambiente térmico (NADER et al., 2002; SAMPAIO et al., 2004; SAMPAIO et al., 2005; SAMPAIO et al., 2007; SILVA et al., 2007, BORGES et al., 2010).

Pesquisas que avaliam o nível de ruído emitido pelos animais em ambiente controlado são recentes e inovadoras, no qual, por intermédio da resposta do grupo de animais, torna-se possível avaliar a situação do ambiente onde estão inseridos. Objetivou-se com este trabalho avaliar a 
influência das variáveis ambientais no nível de ruído emitido por leitões em ambiente controlado e quantificar o nível de ruído em relação às faixas de conforto estabelecidas.

\section{MATERIAL E MÉTODOS}

O experimento foi conduzido em câmara climática, localizada nas dependências do NUPEA Núcleo de Pesquisa em Ambiência, situado junto ao Departamento de Engenharia de Biossistemas/ESALQ - USP, na cidade de Piracicaba - SP.

Foram utilizados cinco leitões em idade de creche (35 dias) para coleta das variáveis a serem analisadas, e, para alocá-los, foi construído um boxe e instalado no interior da câmara.

Para a elaboração do experimento, uma vasta busca na literatura foi realizada, e houve dificuldades em encontrar trabalhos científicos envolvendo animais em ambiente controlado e coleta de dados sonoros. Portanto, para a condução do experimento, uma metodologia teve de ser estabelecida e padronizada, para que o estudo com níveis de ruído animal e variáveis ambientais pudesse ser conduzido. Adotando as faixas de conforto já existentes na literatura para as condições tropicais, os leitões foram submetidos à variação na temperatura de $20^{\circ} \mathrm{C} \mathrm{a} 38^{\circ} \mathrm{C}$, sendo preconizada a elevação da temperatura em $2^{\circ} \mathrm{C}$ a cada 1 hora de exposição dos animais. A umidade do ar variou de acordo com o ar no interior da câmara climática, permanecendo na faixa de $50 \%$ a $80 \%$. O experimento foi realizado em um único dia, totalizando $10 \mathrm{~h}$ de experimentação com os animais.

Para a definição da faixa de temperatura a que os leitões foram submetidos, adotaram-se, como referência, os limites de temperatura e umidade do ar definidos para a situação de conforto e estresse (SILVA, 1999). A faixa de $20^{\circ} \mathrm{C}$ a $23^{\circ} \mathrm{C}$ foi definida como conforto, e temperatura acima de $30^{\circ} \mathrm{C}$ como estresse térmico para leitões com 35 dias de idade, considerando a umidade do ar na faixa de 50\% a 70\%. Para controlar o ambiente interno à câmara climática, havia um dispositivo utilizado para acionar o sistema de aquecimento e/ou resfriamento interno, baseando-se na variação da temperatura e da umidade internas.

A entalpia do ambiente foi calculada de acordo com a eq. (1) proposta por RODRIGUES et al. $(2010)^{1}$, sendo exigência de conforto térmico de leitões em fase de creche, com 35 dias de idade, a faixa de $48-53 \mathrm{~kJ} . \mathrm{kg}^{-1}$ ar seco, e para situação de estresse térmico, valores acima de $88 \mathrm{~kJ}^{-\mathrm{kg}^{-1}}$ ar seco.

$$
h=1,006 \cdot t+\frac{U R}{p_{B}} \cdot 10^{\left(\frac{7,5 \cdot t}{237,3+t}\right)} \cdot(71,28+0,052 . t)
$$

em que,

$\mathrm{h}$ - entalpia, $\mathrm{kJ} \mathrm{kg}^{-1}$ ar seco;

$\mathrm{t}$ - temperatura de bulbo seco do ar, ${ }^{\circ} \mathrm{C}$;

UR - umidade relativa, \%, e

$\mathrm{p}_{\mathrm{B}}$ - pressão barométrica local, $\mathrm{mmHg}$.

\footnotetext{
${ }^{1}$ A equação para cálculo da entalpia referenciada por ROGRIGUES et al. (2010), trata-se de uma recente adaptação da equação proposta por ALBRIGHT (1990), para que valores de temperatura, umidade relativa e pressão atmosférica local possam ser incorporados diretamente na equação para o cálculo da Entalpia.
} 
O tamanho da amostra de animais foi definida com base na área total disponível para alojamento dos leitões no interior da câmara climática. A câmara climática possui uma área interna de aproximadamente $9 \mathrm{~m}^{2}$, sendo 3,85 $\mathrm{m}$ de comprimento por 2,85 $\mathrm{m}$ de largura, pé-direito de 2,90 m. As paredes são revestidas com isolante térmico (PVC), incluindo o teto, o piso de cerâmica e tanto a porta quanto a fonte emissora de calor para dentro da câmara são de aço inoxidável. No centro da câmara climática, foi instalado um boxe de $2 \mathrm{~m}^{2}$ para abrigar os leitões, com dimensões de $1 \mathrm{~m}$ de largura, $2 \mathrm{~m}$ de comprimento e $1,20 \mathrm{~m}$ de altura, sendo disponibilizada uma área mínima de 0,4 $\mathrm{m}^{2}$ por animal, sendo a área mínima recomendada de 0,30 a 0,35 m² (EMBRAPA, 2003).

O piso do boxe foi coberto com maravalha (cama de serragem) para melhorar a aderência dos leitões ao piso, e dentro do boxe foram instalados um comedouro e um bebedouro (FIGURA 1).
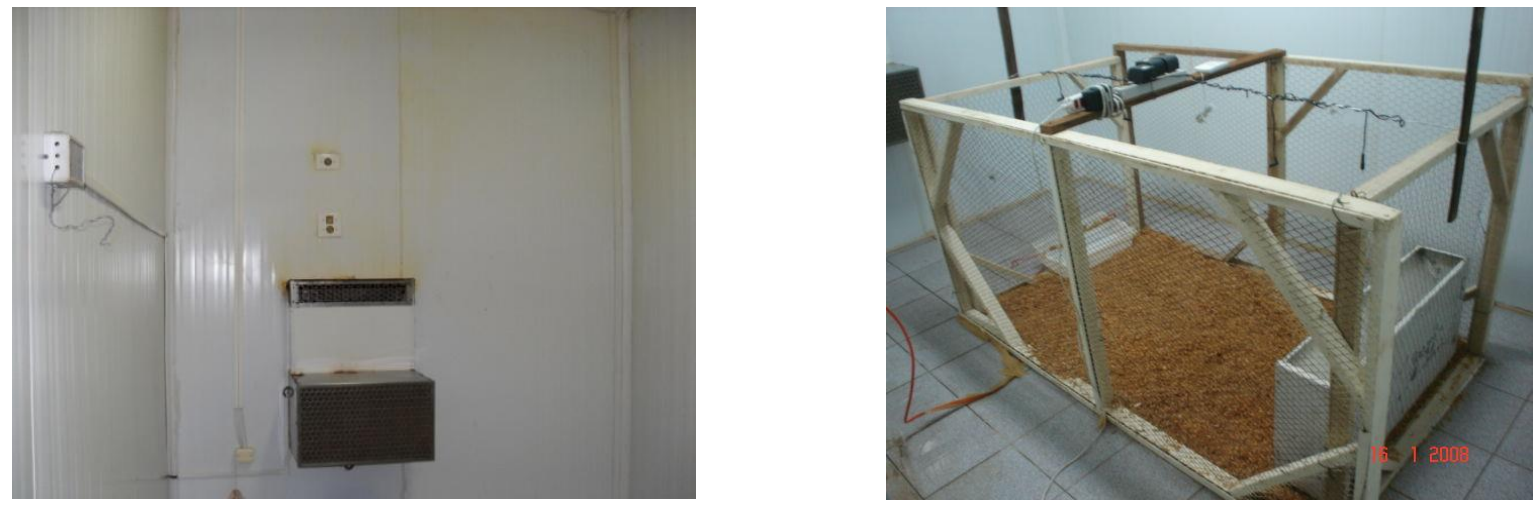

FIGURA 1. Vista interna da câmara climática com o boxe pronto e instalado para receber os leitões e instalar os equipamentos. Inside the environmental control room (left side). Pen used for housing piglets during the trial (right side).

Foram utilizados no experimento, cinco leitões da linhagem landrace x large white, com 35 dias de vida, pesos semelhantes e oriundos de um mesmo lote. Foi adotado um período de adaptação de 5 dias para os leitões em câmara climática. Devido ao número limitado de animais para este estudo prévio, o delineamento experimental foi caracterizado como não estruturado, sem tratamento e/ou estrutura de parcelas. A unidade experimental considerada foi o boxe com os leitões, e os dados coletados a cada 10 minutos foram considerados como medidas repetidas.

Para o registro das variáveis ambientais (temperatura ambiente, ${ }^{\circ} \mathrm{C}$, e umidade do ar, \%), foram instalados dois sensores dataloggers (HOBO, H08007IS) posicionados no centro do boxe, a $1 \mathrm{~m}$ de altura dos animais, com intervalo de registro a cada $10 \mathrm{~min}$.

Para captar os níveis de ruídos emitidos pelos leitões no interior da câmara climática, foram instalados três decibelímetros dataloggers (MINIPA, MSL1352C), que são equipamentos específicos para a captação do nível de ruído, em decibéis $(\mathrm{dB})$. Os decibelímetros foram instalados no centro do boxe contendo os leitões, com o microfone voltado para baixo, a $1 \mathrm{~m}$ de altura do piso. Fontes de energia para manter os equipamentos funcionando foram instaladas e fixadas na parte superior do boxe dos leitões, conforme ilustrado nas FIGURAS 2a e 2b, a seguir. 


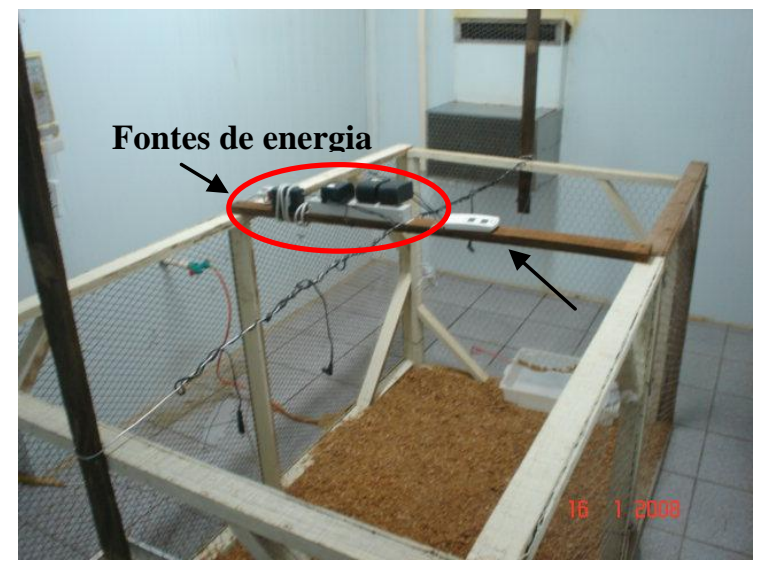

(a)

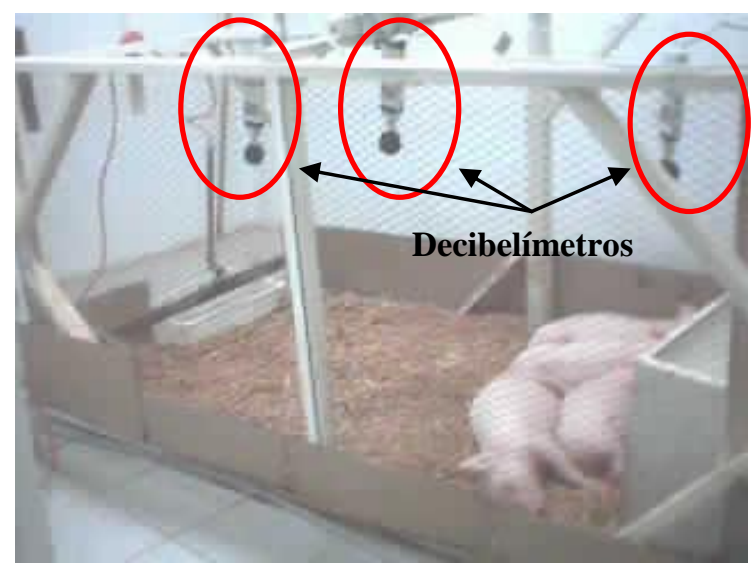

(b)

FIGURA 2. (a) Fontes para energizar os decibelímetros. (b) Locação dos decibelímetros no interior da câmara climática. (a) Power to supply the sound meter levels. (b) Location of the sound level meters inside the environmental control room.

Os dados de ruído foram registrados a cada minuto, e os decibelímetros foram programados para captá-los utilizando o nível de ponderação "C", pois, de acordo com GERGES (2000), essa ponderação é considerada para aferir níveis de ruído do ambiente, sem utilização de filtros, podendo captar sons de baixa frequência. O nível "A" é mais utilizado para simular o que é audível pelo ouvido humano, não sendo, portanto o objeto de estudo deste trabalho. A escala do nível sonoro adotada foi a automática, na faixa de $30 \mathrm{~dB}$ a $130 \mathrm{~dB}$, e a seleção do tempo de resposta da leitura adotada foi o modo "SLOW", pois capta os níveis de ruídos contínuos ou intermitentes, conforme discrimina a Norma Regulamentadora NR -15 (BRASIL, 1978).

A subtração do ruído de fundo foi conduzida, repetindo-se a metodologia acima descrita após a retirada dos leitões, e este foi subtraído do ruído total (leitões + ambiente), restando somente o nível de ruído emitido pelos leitões. Para o cálculo, utilizou-se a eq. (2) descrita por GERGES (2000), apresentada abaixo:

$$
\mathrm{NR}=10 \log \left(10^{\frac{\mathrm{L}_{\mathrm{t}}}{10}}-10^{\frac{\mathrm{L}_{\mathrm{f}}}{10}}\right)
$$

em que,

$\mathrm{NR}$ - Nível de ruído dos leitões (dB);

$\mathrm{L}_{\mathrm{t}}-$ Nível de ruído total $(\mathrm{dB})$;

$\mathrm{L}_{\mathrm{f}}-$ Nível de ruído de fundo $(\mathrm{dB})$.

Para avaliar o nível de atividade motora, foram utilizadas técnicas de processamento de uma sequência de imagens dos leitões, confinados na câmara climática. As imagens foram registradas utilizando uma webcam instalada em uma das laterais da câmara climática.

As imagens foram capturadas a cada 10 minutos e armazenadas utilizando o próprio software do fabricante da câmera, para posterior pré-processamento. Para o pré-processamento das imagens, foram utilizadas as técnicas de limiarização e binarização, que consistem em converter imagens em tons de cinza para imagens binárias, utilizando o Image Processing Toolboxe MATLAB ${ }^{\circledR} 2006 a$. Dessa forma, as imagens foram todas binarizadas e subtraídas duas a duas para detecção de movimento entre as imagens, como ilustra a FIGURA 3. 


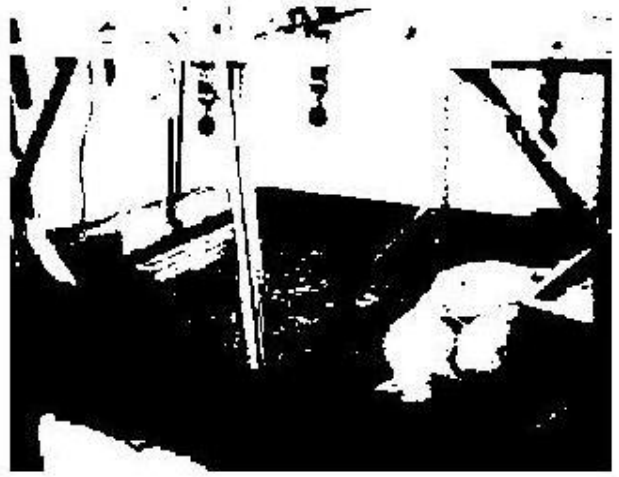

(a)

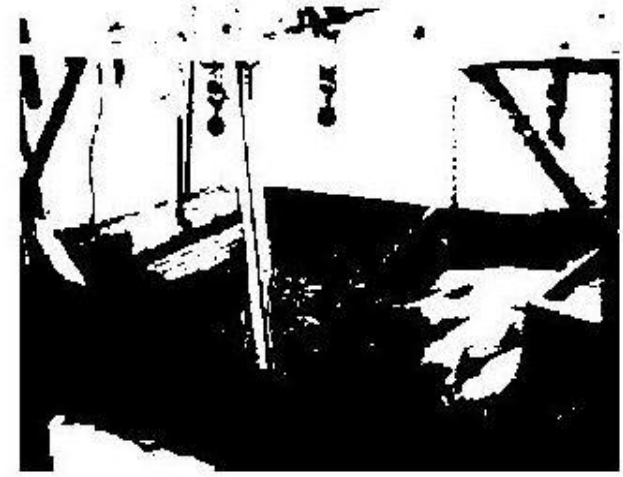

(b)

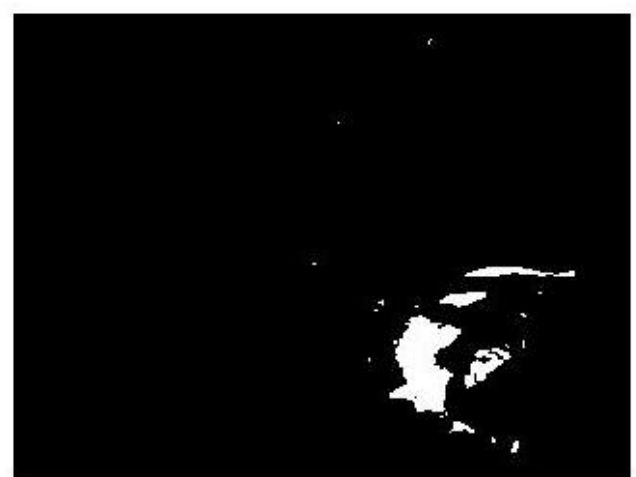

(c)

FIGURA 3. Etapas de utilização das técnicas de binarização e limiarização para posterior análise das imagens e como foi executada a subtração das imagens; (a) imagem posterior; (b) imagem anterior, (c); a diferença entre as imagens, indicando a movimentação dos leitões. Subtract difference between two images. (a) Current image, (b) previously image, (c) subtracting between images resulting the piglets' activity level.

Assim, tem-se que $\mathrm{R}$ é o nível de atividade que varia de 0 a 1 . Para as análises estatísticas da emissão dos níveis de pressão sonora dos leitões em relação às variáveis ambientais (temperatura e umidade do ar), à entalpia e ao nível de atividade motora, foram utilizados teste de correlação e análises de regressão. Foram empregadas as rotinas de procedimento de correlação (procCORR) e de regressão (procREG) do software estatístico SAS ${ }^{\circledR}$ (SAS, 2004).

\section{RESULTADOS E DISCUSSÃO}

A variação dos dados apresentou decréscimo entre os níveis de ruído emitidos pelos leitões tanto para a variável ambiental temperatura quanto para o índice entalpia de conforto. $\mathrm{O}$ índice entalpia de conforto quantificou a energia térmica contida no interior da câmara climática em função das variáveis climáticas, temperatura de bulbo seco e umidade do ar (FIGURA 4).

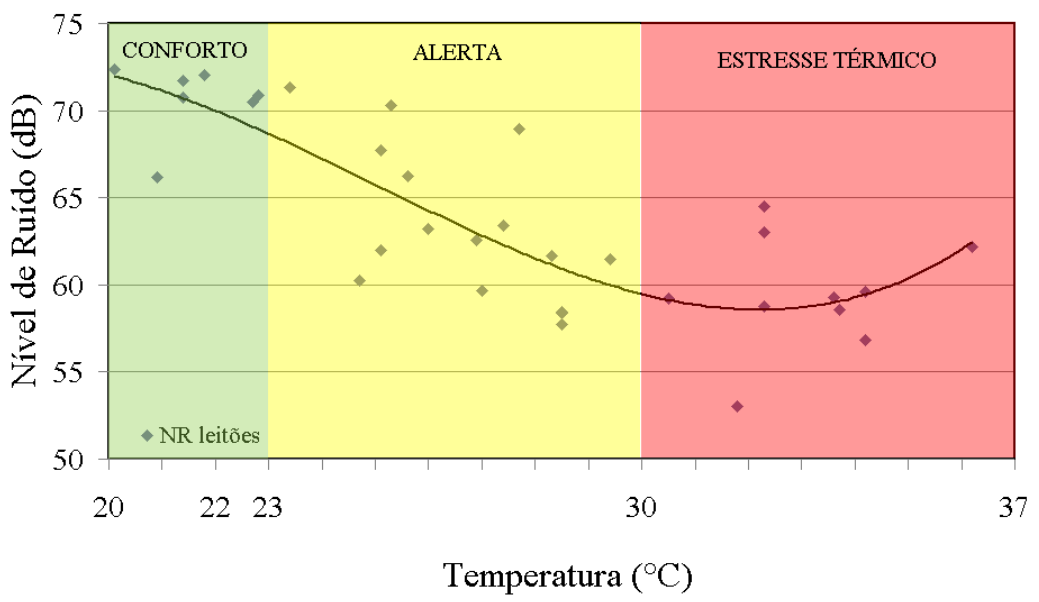




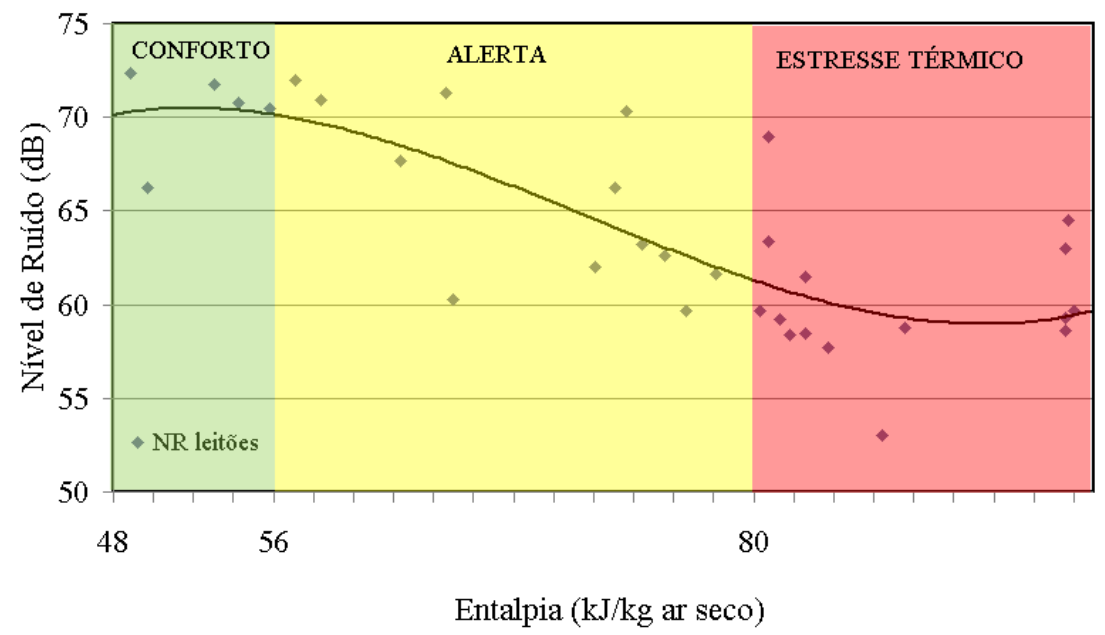

FIGURA 4. Dispersão dos níveis de ruído emitidos pelos suínos em relação à temperatura do ambiente e ao índice entalpia de conforto. Sound level emitted by piglets in relation to temperature (left side) and enthalpy (right side).

Os dados da TABELA 1 mostram que o nível de ruído emitido pelos leitões em condições de estresse térmico foi menor quando comparado à condição de conforto.

TABELA 1. Níveis sonoros (dB) emitidos pelos leitões em diferentes condições térmicas. Sound levels (dB) emitted by piglets at different thermal conditions.

\begin{tabular}{cccc}
\hline & $\begin{array}{c}\text { ENTALPIA } \\
(\mathrm{kJ} / \mathrm{kg} \text { ar } \\
\text { seco })\end{array}$ & $\begin{array}{c}\text { CONDIÇÃO DO } \\
\text { ANIMAL }\end{array}$ & $\begin{array}{c}\text { NÍVEL DE RUÍDO } \\
\text { EMITIDO PELOS } \\
\text { LEITÕES }\end{array}$ \\
\hline $20-23^{\circ} \mathrm{C}$ & $48-56$ & CONFORTO & $70-75 \mathrm{~dB}$ \\
$23-30^{\circ} \mathrm{C}$ & $56-80$ & ALERTA & $60-70 \mathrm{~dB}$ \\
$>30^{\circ} \mathrm{C}$ & $>80$ & ESTRESSE & \\
\hline
\end{tabular}

Os resultados apresentados corroboram SAMPAIO et al. (2007) que, em estudo em ambiente de produção intensiva de suínos, constataram uma intensidade de $65 \mathrm{~dB}$ em instalações de creche. Comparado ao presente estudo, essa intensidade sonora se enquadraria em um quadro de alerta térmico na instalação. Os autores ainda ressaltam que as maiores intensidades sonoras foram encontradas em condições de desconforto térmico para os animais.

De acordo com o gráfico da FIGURA 5, foi possível perceber que os dados se relacionaram, em sua maior parte, com a faixa considerada de alerta por estresse térmico, ou seja, na transição entre o conforto e o estresse calórico. Para a faixa de conforto $\left(20\right.$ a $\left.23^{\circ} \mathrm{C}\right)$ verifica-se que o nível de atividade dos suínos se manteve em valores próximos de zero, ou seja, de baixa movimentação pelos leitões enquanto estes estavam sob condições de conforto térmico. Com a elevação gradativa da temperatura no interior da câmara, os leitões apresentaram comportamento agitado, seguido de brigas, disputa pelo espaço próximo ao bebedouro, inquietação, ocorrendo na faixa de temperatura de 23 a $30^{\circ} \mathrm{C}$. Ao atingir $38^{\circ} \mathrm{C}$, os animais apresentaram-se dispostos, em sua totalidade, próximo ao bebedouro e com sinais críticos de prostração, portanto este fator foi determinante para explicar a declinação da curva do nível de atividade dos leitões. 


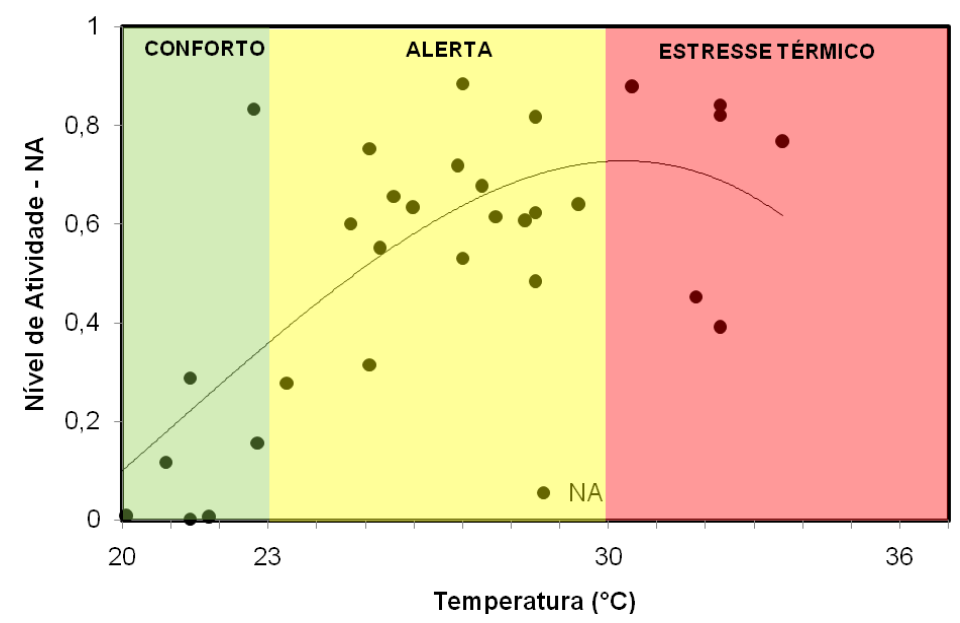

FIGURA 5. Dispersão dos Níveis de Atividade dos suínos em relação à temperatura ambiente. Piglets' activity level compared to thermal conditions.

Os leitões apresentaram tendência de emitirem menos som quando em desconforto térmico, porém sua movimentação na baia foi intensa, devido à necessidade de procura por um local que facilitasse as trocas por condução, devido à dificuldade em efetuar trocas térmicas por evaporação (ESMAY, 1978).

Foram feitas análises de correlação das variáveis coletadas em câmara climática, utilizando a rotina de procedimento de correlação (procCORR) do software estatístico SAS ${ }^{\circledR}$ (SAS, 2004).

Os dados analisados foram coletados a cada $10 \mathrm{~min}$ para as variáveis temperatura $\left(\mathrm{T},{ }^{\circ} \mathrm{C}\right) \mathrm{e}$ umidade do ar (UR, \%) do ambiente, e a entalpia foi calculada em função destas variáveis (RODRIGUES et al., 2010). Os níveis de ruído emitidos pelos leitões $\left(\mathrm{NR}_{\text {leitões }}\right)$ foram captados a cada minuto, porém, para a análise, utilizou-se o dado pontual referente a cada 10 min de dados registrados. Foram obtidos dados da variável resposta (níveis de ruído) e das variáveis explicativas (temperatura e umidade). Após a organização dos dados, estes foram ordenados em função das temperaturas coletadas no interior da câmara climática e assim analisados. Foram feitas análises de correlação de Pearson $(\mathrm{P}<0,05)$, entre as variáveis acima mencionadas e estes, sendo apresentados na TABELA 2.

TABELA 2. Níveis de correlação entre as variáveis explicativas (temperatura, umidade do ar, entalpia e nível de atividade) e a variável resposta nível de ruído emitido pelos leitões. Correlation values among variables (temperature, relative humidity, enthalpy and activity level) and piglets' sound level.

\begin{tabular}{cccccc}
\hline & $\mathrm{NR}_{\text {leitões }}$ & $\mathrm{T}$ & $\mathrm{UR}$ & Entalpia & $\mathrm{NA}$ \\
\hline $\mathrm{NR}_{\text {leitões }}$ & 1 & & & \\
$\mathrm{~T}$ & $-0,7825^{* *}$ & 1 & & \\
& $<10^{-4}$ & & & \\
$\mathrm{UR}$ & $0,3317^{*}$ & $-0,5932^{* *}$ & 1 & \\
& 0,0449 & $<10^{-4}$ & & 1 \\
Entalpia & $-0,7927^{* *}$ & $0,9739^{* *}$ & $-0,4166^{*}$ & \\
& $<10^{-4}$ & $<10^{-4}$ & 0,0103 & \\
$\mathrm{NA}$ & $-0,6427^{* *}$ & $0,7244^{* *}$ & $-0,1107 \mathrm{NS}$ & $0,7773^{* *}$ & 1 \\
& $<10^{-4}$ & $<10^{-4}$ & 0,5141 & $<10^{-4}$ & \\
\hline
\end{tabular}

NS = não significativo, ${ }^{*}$ significativo ao nível de 5\% de probabilidade, $* *$ significativo ao nível de $1 \%$ de probabilidade.

O principal objetivo desta análise é determinar a dependência funcional da variável resposta sobre as variáveis preditoras, ou seja, foi verificar o comportamento do nível de ruído emitido pelos 
leitões frente às variáveis ambientais, índice de conforto e o nível de atividade. Os resultados da tabela mostram que estes valores apresentaram correlação negativa de $-0,7825(\mathrm{P}<0,0001)$ com a temperatura, ou seja, a elevação da temperatura promoveu uma redução dos níveis sonoros emitidos pelos leitões, sendo que $78 \%$ dos dados analisados são explicados pelo efeito da variável temperatura.

Com relação à umidade do ar, o nível de ruído apresentou correlação positiva de $0,3317(\mathrm{P}=$ 0,0449), porém, $33 \%$ dos dados analisados são explicados pelo efeito da variável umidade do ar.

Os resultados divergem de NADER et al. (2002) e SILVA et al. (2007) que, em estudos prévios sobre ruído emitido por suínos, não encontraram correlação entre as variáveis ambientais e os níveis de ruído emitidos por suínos, sendo ambos os trabalhos executados em ambiente de produção intensiva. Os resultados também divergem dos encontrados por SAMPAIO et al., 2007.

O índice de conforto entalpia, seguiu a tendência da temperatura em relação aos níveis de ruído, apresentando uma correlação negativa de $-0,793(\mathrm{P}<0,0001)$. Isso significa que $79 \%$ dos dados analisados são explicados pelo efeito da entalpia no interior da câmara. Os leitões apresentaram estado elevado de prostração, quando em temperaturas acima do limite crítico superior $\left(30^{\circ} \mathrm{C}\right)$, contribuindo para a redução da emissão de sons por parte dos animais.

O nível de atividade apresentou perfil semelhante ao da temperatura e entalpia, com correlação negativa de $-0,64(\mathrm{P}<0,0001)$ entre o nível de ruído emitido dos leitões.

Os níveis de ruído emitidos pelos leitões foram analisados pela estatística de regressão em função das variáveis ambientais, temperatura e umidade do ar e do índice de conforto entalpia, para a estimativa de um modelo de regressão da variável resposta $\left(\mathrm{NR}_{\text {leitões }}\right)$ em relação às variáveis explicativas ( $\mathrm{T}$ e UR). Para as análises, foi utilizada a rotina de procedimento $R E G$ do software estatístico SAS ${ }^{\circledR}$ (SAS, 2004). Os dados foram analisados com melhor ajuste do modelo de regressão cúbica, por apresentarem os maiores coeficientes de determinação.

Os níveis de ruído foram mais bem representados pela temperatura, visto que a regressão simples com relação a esta variável apresentou coeficiente de determinação ajustado ( $\mathrm{R}^{2}$ ajustado) de maior valor, quando comparado com o mesmo para a entalpia.

TABELA 3. Modelos de regressão para a estimativa do nível de ruído dos leitões. Regression models to predict sound levels emitted by piglets.

\begin{tabular}{cccc}
\hline Modelo & $\operatorname{Pr}>\mathrm{F}$ & $\mathrm{R}^{2}$ & $\mathrm{R}^{2}$ ajustado \\
\hline $\mathrm{NPS}_{\text {leitões }}=0,0130 \mathrm{~T}^{3}-1,023 \mathrm{~T}^{2}+25,206 \mathrm{~T}-128,138$ & $<0,0001^{* *}$ & 0,90 & 0,88 \\
$\mathrm{NPS}_{\text {leitões }}=-0,003 \mathrm{UR}^{3}+0,703 \mathrm{UR}^{2}-50,345 \mathrm{UR}+1227,156$ & $0,138 \mathrm{NS}$ & 0,38 & 0,21 \\
$\mathrm{NPS}_{\text {leitões }}=0,0004 \mathrm{H}^{3}-0,084 \mathrm{H}^{2}+5,796 \mathrm{H}-55,885$ & $<0,0001^{* *}$ & 0,89 & 0,86 \\
\hline
\end{tabular}

NS = não significativo, ${ }^{* *}$ significativo ao nível de $1 \%$ de probabilidade.

Em estudos prévios, SILVA et al. (2007) avaliaram o nível de ruído de leitões em creche convencional e encontraram valores médios de $12,49 \mathrm{~dB}$, não encontrando correlação entre o ambiente térmico da instalação. $\mathrm{O}$ valor médio encontrado resultou da subtração entre o ruído total (animal + meio) e o ruído de fundo (meio), porém a subtração não foi realizada conforme recomendado por GERGES (2000), sendo utilizados dados de maneira errônea, para análise. Utilizando os dados de ruído total do presente trabalho, foram encontrados valores de nível de ruído na faixa de 72 a $76 \mathrm{~dB}$, que quando comparado aos valores propostos na TABELA 1, o nível de ruído enquadra-se em uma condição de conforto para os leitões alojados.

\section{CONCLUSÕES}

As variáveis ambientais influenciam na emissão de ruído pelos leitões quando expostos a diferentes condições térmicas. 
Os níveis de ruídos foram estabelecidos em faixas de acordo com a condição térmica a que os animais foram submetidos. Para a condição de conforto $\left(20\right.$ a $\left.23^{\circ} \mathrm{C}\right)$, níveis de ruído na faixa de 70 a $75 \mathrm{~dB}$; condição de alerta $\left(23\right.$ a $\left.30^{\circ} \mathrm{C}\right)$, níveis de ruído na faixa de 60 a $70 \mathrm{~dB}$, e para condição de estresse térmico (acima de $30^{\circ} \mathrm{C}$ ), na faixa de 55 a $60 \mathrm{~dB}$.

\section{REFERÊNCIAS}

ALBRIGHT, L.D. Environment control for animals and plants. St Joseph: ASAE, 1990. 453p. (ASAE Textbook, 4).

BANHAZI, T.; AARNINK, A.; THUY, H.; PEDERSEN, S.; HARTUNG, J.; PAYNE, H.; MULLAN, B.; BERCKMANS, D. Review of issues related to heat stress in intensively housed pigs. In: INTERNATIONAL LIVESTOCK ENVIRONMENT SYMPOSIUM, 8., 2008, Foz do Iguaçu. Proceedings... Foz do Iguaçu: ASABE, 2008. 1 CD-ROM.

BARBOSA FILHO, J.A.D.; SILVA, I.J.O.; SILVA, M.A.N.; SILVA, C.J.M. Avaliação dos comportamentos de aves poedeiras utilizando sequência de imagens. Engenharia Agrícola, Jaboticabal, v.27, n. 1, p. 93-99, 2007.

BOISSY, A.; MANTEUFFEL, G.; JENSEN, M.B.; MOE, R.O.; SPRUIJT, B.; LINDA J.; KEELING, L. J.; WINCKLER, C.; FORKMAN, B.; DIMITROV, I.; LANGBEIN, J.; BAKKEN, M.; VEISSIER, I.; AUBERT, A. Assessment of positive emotions in animals to improve their welfare. Physiology \& Behavior, Elmsford, v. 92, p. 375-397, 2007.

BORGES, G.; MIRANDA, K.O.S.; RODRIGUES, V.C.; RISI, N. Uso da geoestatística para avaliar a captação automática dos níveis de pressão sonora em instalações de creche para suínos. Engenharia Agrícola, Jaboticabal, v.30, n. 3, p. 377-385, 2010.

BRASIL. Ministério do Trabalho e Emprego. Portaria 3.214, de jul. 1978. Normas regulamentadoras de segurança e saúde no trabalho - NR-15: atividades e operações insalubres. Brasília, 1978.

BROWN-BRANDL, T.M.; EIGENBERG, R.A.; NIENABER, J.A.; KACHMAN, S.D. Thermoregulatory profile of a newer genetic line of pigs. Livestock Production Science, Amsterdam, v. 71, p. 253- 260, 2001.

EMBRAPA. EMPRESA BRASILEIRA DE PESQUISA AGROPECUÁRIA. Sistema de produção de suínos. 2003. Disponível em: <http://sistemasdeproducao.cnptia.embrapa.br/FontesHTML /Suinos/SPSuinos/construcao.html>. Acesso em: 12 dez. 2011

ESMAY, M.L. Principles of animal environment. West Port: Avi Publication, 1978. 325 p.

GERGES, S.N.Y. Ruído: fundamentos e controle. 2.ed. Florianópolis: DEM, UFSC, 2000. 696 p.

HUYNH, T. T. T.; AARNINK, A.J.A.; SPOOLDER, H.A.M.; VERSTEGEN, M.W.A.; KEMP, B. Effects of floor cooling during high ambient temperatures on the lying behavior and productivity of growing finishing pigs. Transactions of the ASAE, St. Joseph, v. 47, n. 5, p. 1.773-1.782, 2004.

HUYNH, T.T.T.; AARNINK, A. J. A.; GERRITS, W. J. J.; HEETKAMP, M. J. H.; CANH, T.T.; SPOOLDER, H.A.M.; KEMP, B.; VERSTEGEN, M.W.A. Thermal behavior of growing pigs in response to high temperature and humidity. Applied Animal Behaviour Science, Amsterdam, v. 91, p.1-16, 2005.

MANNO, M. C.; OLIVEIRA, R. F. M.; DONZELE, J. L.; FERREIRA, A. S.; OLIVEIRA, W. P.; LIMA, K. R. S.; VAZ, R. G. M. V. Efeito da Temperatura Ambiente sobre o Desempenho de Suínos dos 15 aos 30 kg. Revista Brasileira de Zootecnia, Viçosa, v. 34, n.6, p. 1963-1970, 2005.

NÄÄS, I.A.; CAMPOS, L.S.L.; BARACHO, M.S.; TOLON, Y.B. Uso das redes neurais artificiais na identificação de vocalização de suínos. Engenharia Agrícola, Jaboticabal, v. 28, n. 2, p. 204-216, abr./jun. 2008. 
NADER, A.S.; BARACHO, M.S.; NÄÄS, I.A.; SAMPAIO, C.A.P. Avaliação dos níveis de ruídos e da qualidade do ar (com relação à presença de gases e fungos) em creche de suínos. In: SEMINÁRIO DE POLUENTES AÉREOS E RUÍDOS EM INSTALAÇÕES PARA A PRODUÇÃO ANIMAL, 2002, Campinas. Anais... Campinas: UNICAMP, 2002. p. 49-56.

PANDORFI, H.; SILVA, I.J.O.; MOURA, D.J.; SEVEGNAN, K.B.N. Análise de imagem aplicada ao estudo do comportamento de leitões em abrigo escamoteador. Engenharia Agrícola, Jaboticabal, v.24, n.2, p.274-284, 2004.

RODRIGUES, V.C. Distribuição espacial e bem-estar de aves poedeiras em condições de estresse e conforto térmico utilizando visão computacional e inteligência artificial. 2006. $101 \mathrm{f}$. Dissertação (Mestrado em Física do Ambiente Agrícola) - Escola Superior de Agricultura "Luiz de Queiroz", Universidade de São Paulo, Piracicaba, 2006.

RODRIGUES, V.C.; SILVA, I.J.O.; VIEIRA, F.M.C.; NASCIMENTO, S.T. A correct enthalpy relationship as thermal comfort index for livestock. International Journal of Biometeorology, Suisse, v. 55, n. 3, p. 455-459, 2010.

ROSNER, S.J.; OWEN, G.; CLIFFORT JÚNIOR, G.M. Method and system for determining motion based on difference image correlation: International Search Report Appln. No. PCT/US05/39493, 2006. Disponível em: <http://www.patentstorm.us/patents/7247836.html>. Acesso em: 19 dez. 2011.

SAMPAIO, C.A.P.; CRISTANI, J.; DUBIELA, J.; BOFF, C.E.; OLIVEIRA, M.A. Avaliação do ambiente térmico em instalação para crescimento e terminação de suínos utilizando os índices de conforto térmico nas condições tropicais. Revista Ciência Rural, Santa Maria, v. 34, n. 3, p. 785790, 2004.

SAMPAIO, C.A.P.; NÄÄS, I.A.; NADER, A.S. Gases e ruídos em edificações para suínos aplicação das normas NR-15, CIGR e ACGIH. Engenharia Agrícola, Jaboticabal, v. 25, n. 1, p. 10$18,2005$.

SAMPAIO, C.A.P.; NÄÄS, I.A.; SALGADO, D.D.; QUEIRÓS, M.P.G. Avaliação do nível de ruído em instalações para suínos. Revista Brasileira de Engenharia Agrícola Ambiental, Campina Grande, v. 11, n. 4, p. 436-440, 2007.

SAS INSTITUTE. SAS OnlineDoc® 9.1.3. Cary, 2004.

SILVA, I.J.O. Sistemas naturais e artificiais do controle do ambiente - Climatização. In: SILVA, I.J.O. Ambiência e qualidade na produção industrial de suínos. Piracicaba: FEALQ, 1999. cap. 7, p. 149-179.

SILVA, K.O.; NÄÄS, I.A. Utilização da vocalização como ferramenta para avaliação do comportamento dos suínos. In: CONGRESSO BRASILEIRO DE BIOMETEOROLOGIA, 4., 2006, Ribeirão Preto. Anais... Ribeirão Preto: Sociedade Brasileira de Biometeorologia, 2006. 1 CD-ROM.

SILVA, K.O.; NÄÄS, I.A.; TOLON, Y.B.; CAMPOS, L.S.L.; SALGADO, D.D. Medidas do ambiente acústico em creche de suínos. Revista Brasileira de Engenharia Agrícola e Ambiental, Campina Grande, v. 11, n. 3, p. 339-344, 2007.

SILVA, W.T.; MOURA, D.J.; NÄÄS, I A.; MENDES, A.S.; LIMA, K.A.O. Estimativa de bem-estar de leitões utilizando a visão computacional. Revista Brasileira de Agroinformática, Lavras, v. 6, n. 1, p. 79-89, 2004.

VAN HIRTUM, A.; BERCKMANS, D. Objective recognition of cough sound as a biomarker for aerial pollutants. Indoor Air, Copenhagen, v. 14, p. 10-15, 2004. 